\title{
Systematic review of mental health comorbidities in psoriatic arthritis
}

\author{
Sizheng Steven Zhao ${ }^{1,2} \cdot$ Natasha Miller ${ }^{1,3} \cdot$ Nicholas Harrison $^{2} \cdot$ Stephen J. Duffield ${ }^{1} \cdot$ Mrinalini Dey $^{1,2}$. \\ Nicola J. Goodson ${ }^{1,2}$
}

Received: 6 June 2019 / Revised: 5 July 2019 / Accepted: 1 August 2019 /Published online: 5 September 2019

(C) The Author(s) 2019

\begin{abstract}
Objective In this systematic review and meta-analysis of psoriatic arthritis (PsA) studies, we pooled data from existing literature to (1) estimate the prevalence of mental health disorders in PsA patients and (2) compare disease activity in PsA patients with and without these comorbidities.

Method We searched PubMED, Web of Science, Scopus, PsycINFO and the Cochrane Library using a predefined protocol in accordance with Preferred Reporting Items for Systematic reviews and Meta-Analyses (PRISMA) guidelines. Where possible, meta-analysis was performed using random effects model. Prevalence estimates were pooled according to the severity of mental health disorders.

Results A total of 24 studies, amounting to 31,227 PsA patients, were included for review. Anxiety and depression were the only consistently reported mental health disorders, defined using a range of screening criteria/thresholds. Anxiety prevalence ranged from 4 to $61 \%$ with a pooled estimate of $33 \%$ (95\%CI 17 to 53\%) having at least mild anxiety and $21 \%$ (95\%CI 14 to $29 \%$ ) at least moderate. Depression prevalence ranged from 5 to $51 \%$, with $20 \%$ (95\% CI 8 to $35 \%$ ) having at least mild and $14 \%$ (95\% CI 8 to $21 \%$ ) at least moderate. Only two studies compared disease activity according to the presence of mental health comorbidities; both reported higher disease activity and pain among those with comorbid anxiety and depression.

Conclusions Anxiety and depression are highly prevalent among PsA patients. Studies of other mental health disorders were scarce. More studies are needed on the impact of these comorbidities on disease activity and long-term outcomes.

Key Points

- One in three patients with psoriatic arthritis has at least mild anxiety, while 1 in 5 reported at least mild depression.

- PsA patients with anxiety and/or depression reported greater disease activity.

- More research is needed on other mental health comorbidities, particularly sleep, suicide/self-harm and substance misuse.
\end{abstract}

Keywords Anxiety $\cdot$ Comorbidity $\cdot$ Depression $\cdot$ Mental health $\cdot$ Meta-analysis $\cdot$ Prevalence $\cdot$ Psoriatic arthritis $\cdot$ Sleep . Systematic review

\section{Background}

Electronic supplementary material The online version of this article (https://doi.org/10.1007/s10067-019-04734-8) contains supplementary material, which is available to authorized users.

Nicola J. Goodson nicola.goodson@liverpool.ac.uk

1 Musculoskeletal Biology I, Institute of Ageing and Chronic Disease, University of Liverpool, Liverpool, UK

2 Department of Academic Rheumatology, Aintree University Hospital, Liverpool, UK

3 School of Medicine, University of Liverpool, Liverpool, UK
Psoriatic arthritis (PsA) affects 0.3 to $1 \%$ of the general population and up to $30 \%$ of those with psoriasis [1]. The articular manifestations can be debilitating, afflicting varying number and distribution of peripheral joints as well as the axial skeleton. In addition, multiple domains of health can be affected by skin involvement, numerous other disease features (e.g. enthesitis, dactylitis, nail disease, uveitis) and additional, coexisting morbidities [2]. Mental health disorders are an important and highly prevalent group of comorbidities in patients with psoriasis [3], but their prevalence and impact in PsA have not been systematically reviewed. Prevalence of these comorbidities may differ between the two disease groups since PsA 
cohorts tend to be older and have a longer duration of skin involvement - the majority of PsA patients have had skin involvement for $\sim 10$ years [1]. Psoriasis patients who develop arthritis have higher levels of fatigue and psychological distress [4]. In patients with PsA, joint involvement appears to affect the quality of life to a greater extent than skin [5].

Improving the understanding of mental health comorbidities in PsA has significant clinical implications. Studies have shown that patients with depression have increased the perception of pain [6]. Poor mental health is also a key predictor for secondary fibromyalgia [7] which, in turn, is associated with worse patient-reported outcomes [8]. These comorbidities present challenges when trying to treat PsA to target. Composite treatment targets, such as DAS28 [9] or minimal disease activity [10], include components (tender joint count, pain and patient global visual analogue scales) that may be influenced by comorbid mental health conditions. Treatment escalation driven by non-inflammatory symptoms may lead to toxicity. This is particularly important in PsA management since tight control was associated with more adverse events than standard care, was not superior for radiological damage, and only provided modest improvements to the quality of life [11].

The aims of this systematic review were to (1) describe the prevalence of comorbid mental health disorders in PsA and (2) compare disease activity scores in PsA patients with and without these comorbidities.

\section{Methods}

We conducted a systematic review in accordance with the Preferred Reporting Items for Systematic Reviews and Meta-Analyses (PRISMA) guidelines [12]. The protocol was registered in advance (PROSPERO: CRD42017079841). We searched PubMED, Web of Science, Scopus, PsycINFO and the Cochrane Library through February 2019. Details of the search strategy are shown in Supplementary Materials.

Studies recruiting adult patients were included if they reported the prevalence of a comorbid mental health disorder or assessed the effect of a comorbid mental health disorder on disease activity. Studies were excluded if they used nonrepresentative sampling (highly selective recruitment criteria, such as studies that only recruited women), had a sample size of less than 30 (to avoid unreliable prevalence estimates) or used the same cohort as an existing study. Only full-length articles were considered; reviews, comments and editorials were excluded.

Two reviewers independently assessed the eligibility of articles for inclusion and extracted data from suitable studies. Disagreements were resolved through discussion moderated by a third reviewer. Information from included studies was extracted into predefined tabulated summaries containing: study design, sample size, criteria used to diagnose the psoriatic arthritis and mental health disorders, age gender and prevalence of comorbid mental health disorders. Where available, disease activity scores for patients with and without comorbid mental health disorders were also extracted.

Commonly used thresholds for each screening tool were applied to categorise severity of depression. For the Hospital Anxiety and Depression Scale (HADS) anxiety and depression subscales, a scale of $\leq 7$ was interpreted as no anxiety/ depression, 8 to 10 as mild, 11 to 14 moderate, and $\geq 15$ severe [13]. The Patient Health Questionnaire (PHQ-9) thresholds were $\leq 5$ no depression, 6 to 10 mild, 11 to 14 moderate and $\geq 15$ severe [14]. Hamilton Depression Scale (HAM-D) is as follows: $\leq 7$ no depression, 8 to 13 mild, 14 to 18 moderate and $\geq 19$ severe depression [15]. For the Generalised Anxiety Disorder questionnaire (GAD-7), a scale of $\leq 5$ was taken to indicate no anxiety, 6 to 9 mild, 10 to 14 moderate and $\geq 15$ severe [16]. Studies that used similar thresholds were grouped together. We also included studies using components from the quality of life questionnaires to screen for anxiety or depression. The 5-domain EuroQol questionnaire (EQ-5D) has a single question with the options: I am not anxious or depressed; I am moderately anxious or depressed; I am extremely anxious or depressed. Although not a validated method, single-item screening questionnaires have good sensitivity and specificity [17]. The Short-Form Health Survey (SF-36) mental health subscale $(\mathrm{MH})$ and mental component summary (MCS) score also perform well as a screening method [18]. The PsA quality of life questionnaire includes one item "I feel there is no enjoyment in my life" [19]. If a study reported depression prevalence using two different thresholds, it could contribute to more than one pooled estimate.

Quality assessment of included studies was performed using the Newcastle-Ottawa Scale (NOS), adapted for crosssectional studies [20]. Articles were assigned up to six stars depending on the rigour of the methodological approach used (see Supplementary Materials for details).

\section{Statistical analysis}

Prevalence was presented as percentages (95\% confidence interval, $I^{2}$ statistic). Proportional meta-analysis was performed using double arcsine transformation [21], with results presented using random effects models (DerSimonian-Laird). Heterogeneity of meta-analysis estimates was presented using the $I^{2}$ statistic. Funnel and Doi plots were used to assess the risk of publication bias [21]. Where available, differences in markers of disease activity and functional impairment were compared between groups with and without depression. Analyses were performed using MetaXL Version 5.3 (Sunrise Beach, Australia; www.epigear.com). 


\section{Results}

The searched returned a total of 1216 unique publications. After screening and full-text assessment, 27 studies met eligibility criteria. Among these, three were excluded as they used the same cohort as other included studies [22-24]. A flowchart of the selection process is shown in Supplementary Fig. 1 .

Details extracted from the 24 included studies are shown in Table 1 . Collectively they assessed the prevalence of comorbid mental health disorders in 31,227 psoriatic arthritis patients. Individual study sample sizes varied from 41 to 4795 . Seventeen studies were crosssectional in design and 7 were longitudinal (including 2 trials). PsA was defined using a number of approaches: the CASPAR criteria was used in 8 studies (only part of the population fulfilled the criteria in 2), 8 relied on physician/rheumatologist diagnosis only, 5 used ICD (International Classification of Diseases) codes, 2 used patients' self-report and 1 used the Moll and Wright criteria.

According to the NOS, the quality of these studies was generally low (range 0 to 4 out of 6 possible stars). Justification of sample size and comparison of patients in/ excluded from analyses were particularly lacking (Supplementary Table 1).

The most commonly studied mental health comorbidities were anxiety and depression. Ten studies used validated questionnaires to define anxiety and/or depression (6 HADS with various thresholds, 2 PHQ-9, 1 GAD-7, 1 HAM-D), 5 used components from other questionnaires (2 EQ-5D, 2 SF-36, 1 PsA-QoL), 6 used ICD codes and 5 self-report. Some studies used more than one method, but none used diagnostic criteria. Two studies reported poor sleep as screened using the Pittsburgh Sleep Quality Index (PSQI) [47, 48]; their pooled prevalence was $84 \%$ (95\%CI 78 to $90 \%, I^{2}=0 \%$ ).

\section{Prevalence of anxiety}

The prevalence of anxiety ranged from 4 to $61 \%$, depending on the HADS thresholds used. Figure 1a shows the prevalence of at least mild anxiety (HADS-A $\geq 8$ ) as 33\% (95\%CI 17 to $53 \%, I^{2}=99 \%$ ) [26-29]. Figure $1 \mathrm{~b}$ shows the pooled prevalence for at least moderate anxiety (HADS-A $\geq 11$ and GAD-7 $\geq 10$ ) as $21 \%$ (95\%CI 14 to $29 \%, I^{2}=96 \%$ ) [25-31]; excluding the single study using GAD-7 [31] did not improve heterogeneity (data not shown). The prevalence of anxiety using ICD-9 codes was $6 \%\left(95 \%\right.$ CI 2 to $\left.12 \%, I^{2}=99 \%\right)[37,38]$. There was major asymmetry in the funnel and Doi plots for both mild and moderate anxiety prevalence estimates (Supplementary Fig. 2).

\section{Prevalence of depression}

The prevalence of depression ranged from 5 to $51 \%$, depending on the thresholds used. The prevalence of at least mild depression (HADS-D $\geq 8$, PHQ-9 $\geq 5$, HAM-D $\geq 7$ ) was $20 \%$ (95\%CI 8 to $35 \%, I^{2}=99 \%$; Fig. 2a) $[26-29$, $31,33]$; keeping only studies using HADS-D did not improve heterogeneity (data not shown). The pooled prevalence of at least moderate depression (HADS-D $\geq 11$, PHQ-9 $\geq 10$ ) was $14 \%$ (95\%CI 8 to $21 \%, I^{2}=96 \%$; Fig. 2b) [25-30, 32]; excluding the single study using PHQ-9 did not improve heterogeneity (data not shown). Three studies reported the prevalence of depression using patient self-reported diagnosis: the pooled estimate was $22 \%$ (95\%CI 9 to $39 \%, I^{2}=98 \%$ ) [40-42]. Only two studies reported depression prevalence using ICD-9 codes: the pooled estimate was $9 \%\left(95 \%\right.$ CI 3 to $\left.17 \%, I^{2}=100 \%\right)$ $[37,38]$. There was minor asymmetry in the funnel and Doi plots for mild depression, but major asymmetry for moderate depression (Supplementary Fig. 3).

\section{Prevalence of anxiety and/or depression}

Eight studies reported the combined prevalence of anxiety and/or depression. Figure 3a shows the pooled prevalence of anxiety or depression as $22 \%$ (95\% CI 7 to $42 \%, I^{2}=$ 99\%) [31, 34, 35, 39, 43, 44]. The prevalence using screening questionnaires was higher than studies using self-report or ICD code. Figure $3 \mathrm{~b}$ shows the pooled prevalence of anxiety and depression as $29 \%$ (95\% CI 20 to $38 \%, I^{2}=94 \%$ ) $[26,28,30,31]$. There was no asymmetry in the funnel or Doi plots (Supplementary Fig. 4). Khraishi et al. reported a higher prevalence of these comorbidities in established than early PsA (17 vs 9.5\%) [44].

\section{Mental health comorbidities and disease activity}

Only two studies compared disease activity scores in patients with and without comorbid mental health disorders. Freire et al. found that DAS28 (3.2 vs $2.9 p<0.05)$ and pain visual analogue scale (VAS) $(4.1$ vs $3.0 p<0.01)$ were higher in patients with anxiety than those without [30]. Similarly, DAS28 and pain VAS were higher in those with depression than those without. BASDAI was only assessed in a small subgroup, thus comparisons were underpowered. Michelsen et al. compared those with and without anxiety or depression [35]. In addition to DAS28 and pain VAS, they also found differences in the tender joint count, patient and evaluators global, but not swollen joint count, ESR or CRP (Table 2). 
Table 1 Summary of study characteristics, prevalence of depression and quality of studies included in this meta-analysis

\begin{tabular}{|c|c|c|c|c|c|c|c|c|}
\hline Study & $\begin{array}{l}\text { Study } \\
\text { design }\end{array}$ & PsA definition & $\begin{array}{l}\text { Sample } \\
\text { size }\end{array}$ & $\begin{array}{l}\text { Age, } \\
\text { mean } \\
(\mathrm{SD})\end{array}$ & $\begin{array}{l}\text { Females, } \\
\%\end{array}$ & $\begin{array}{l}\text { Mental health } \\
\text { comorbidity }\end{array}$ & $\begin{array}{l}\text { Prevalence, } \\
\%\end{array}$ & $\begin{array}{l}\text { Criteria or definition } \\
\text { used }\end{array}$ \\
\hline $\begin{array}{l}\text { Cauli (2011) } \\
\text { [25] }\end{array}$ & $\mathrm{CS}$ & CASPAR & 319 & $52(13)$ & 42 & $\begin{array}{l}\text { Anxiety } \\
\text { Depression }\end{array}$ & $\begin{array}{l}13.4 \\
11.6\end{array}$ & $\mathrm{HADS} \geq 11$ \\
\hline $\begin{array}{l}\text { McDonough } \\
\text { (2014) [26] }\end{array}$ & $\mathrm{CS}$ & CASPAR & 306 & $54(13)$ & 39 & $\begin{array}{l}\text { Anxiety (HADS-A } \geq 8) \\
\text { Anxiety (HADS-A } \geq 11) \\
\text { Depression }(H A D S-D \geq 8) \\
\text { Depression (HADS-D } \geq 11 \text { ) } \\
\text { Anxiety and depression } \\
\text { (HADS-A and -D } \geq 8 \text { ) } \\
\text { Anxiety and depression } \\
\text { (HADS-A and -D } \geq 11)\end{array}$ & $\begin{array}{l}36.6 \\
15.0 \\
22.3 \\
9.2 \\
17.7 \\
\\
3.9\end{array}$ & HADS \\
\hline $\begin{array}{l}\text { Gniadecki } \\
\quad \text { (2012) [27] }\end{array}$ & $\mathrm{RCT}$ & $\begin{array}{l}\text { Diagnosed by } \\
\text { rheumatologist, } \\
\text { trained assessor or } \\
\text { dermatologist }\end{array}$ & 752 & 47 & 37 & $\begin{array}{l}\text { Anxiety }(\text { HADS-A } \geq 8) \\
\text { Anxiety }(H A D S-A \geq 11) \\
\text { Depression }(H A D S-D \geq 8) \\
\text { Depression }(H A D S-D \geq 11)\end{array}$ & $\begin{array}{l}54 \\
30 \\
37 \\
16\end{array}$ & HADS \\
\hline $\begin{array}{l}\text { Meesters } \\
\quad(2014)[28]\end{array}$ & $\mathrm{CS}$ & ICD-10 codes & 1185 & $58(14)$ & 58 & $\begin{array}{l}\text { Anxiety (HADS-A } \geq 8 \text { ) } \\
\text { Anxiety (HADS-A } \geq 11 \text { ) } \\
\text { Anxiety (HADS-A } \geq 15) \\
\text { Depression (HADS-D } \geq 8 \text { ) } \\
\text { Depression (HADS-D } \geq 11 \text { ) } \\
\text { Depression (HADS-D } \geq 15 \text { ) } \\
\text { Anxiety and depression } \\
\text { (HADS-A and -D } \geq 8 \text { ) }\end{array}$ & $\begin{array}{l}30.5 \\
13.5 \\
4.0 \\
14.8 \\
4.5 \\
0.9 \\
11.2\end{array}$ & HADS \\
\hline $\begin{array}{l}\text { Howell } \\
\text { (2018) [29] }\end{array}$ & $\mathrm{CS}$ & $\begin{array}{l}\text { Self-report of physician } \\
\text { diagnosis }\end{array}$ & 179 & $45(10)$ & 77 & $\begin{array}{l}\text { Anxiety }(\text { HADS-A } \geq 8) \\
\text { Anxiety }(\text { HADS-A } \geq 11) \\
\text { Depression }(H A D S-D \geq 8) \\
\text { Depression }(H A D S-D \geq 11)\end{array}$ & $\begin{array}{l}60.8 \\
40.9 \\
51.2 \\
28.2\end{array}$ & HADS \\
\hline $\begin{array}{l}\text { Freire (2011) } \\
\quad[30]\end{array}$ & $\mathrm{CS}$ & $\begin{array}{l}\text { Rheumatologist } \\
\text { diagnosis }\end{array}$ & 495 & $50(13)$ & 43 & $\begin{array}{l}\text { Anxiety } \\
\text { Depression } \\
\text { Anxiety and depression }\end{array}$ & $\begin{array}{l}29.7 \\
17.6 \\
14.3\end{array}$ & $\begin{array}{l}\text { HADS } \geq 11 \text { or receiving } \\
\text { pharmacological } \\
\text { treatment }\end{array}$ \\
\hline $\begin{array}{l}\text { Lamb (2017) } \\
\quad[31]\end{array}$ & $\mathrm{CS}$ & $\begin{array}{l}\text { Rheumatologist } \\
\text { diagnosis }\end{array}$ & 109 & NS & NS & $\begin{array}{l}\text { Anxiety (GAD-7 } \geq 10 \text { ) } \\
\text { Anxiety (GAD-7 } \geq 15 \text { ) } \\
\text { Depression (PHQ-9 } \geq 5 \text { ) } \\
\text { Depression (PHQ-9 } \geq 20 \text { ) } \\
\text { Anxiety or depression } \\
\text { Anxiety and depression }\end{array}$ & $\begin{array}{l}13.1 \\
8.1 \\
9.9 \\
4.1 \\
14.8 \\
8.1\end{array}$ & $\begin{array}{l}\text { GAD-7 } \\
\text { PHQ-9 } \\
\text { GAD-7/PHQ-9 }\end{array}$ \\
\hline $\begin{array}{l}\text { Kotsis (2012) } \\
\quad[32]\end{array}$ & $\mathrm{CS}$ & CASPAR & 83 & $49(12)$ & 47 & Depression & 21.7 & $\begin{array}{l}\text { PHQ-9 } \geq 10 \text { (at least } \\
\text { moderate) }\end{array}$ \\
\hline $\begin{array}{l}\text { Papp (2014) } \\
{[33]}\end{array}$ & RCT & Physician diagnosis & 523 & 47 (13) & 33 & Depression & 32.4 & $\begin{array}{l}\mathrm{HAM}-\mathrm{D} \geq 7 \text { (at least } \\
\text { mild) }\end{array}$ \\
\hline $\begin{array}{l}\text { Torre-Alonso } \\
\text { (2014) [34] }\end{array}$ & $\mathrm{L}$ & Moll \& Wright & 197 & 4912 & 42 & Anxiety or depression & 50 & EQ-5D \\
\hline $\begin{array}{l}\text { Michelsen } \\
\text { (2017) [35] }\end{array}$ & $\mathrm{L}$ & $\begin{array}{l}\text { Rheumatologist } \\
\text { diagnosis }\end{array}$ & 728 & $48(13)$ & 51 & Anxiety or depression & $\begin{array}{l}44.8 \\
15.9 \\
25.7\end{array}$ & $\begin{array}{l}\text { EQ-5D-3L } \\
\text { SF-36 MH } \leq 56 \\
\text { SF-36 MCS } \leq 38\end{array}$ \\
\hline $\begin{array}{l}\text { Walsh (2014) } \\
\quad[36]\end{array}$ & $\mathrm{CS}$ & $\begin{array}{l}\text { Rheumatologist } \\
\text { diagnosis, 93\% } \\
\text { fulfilled CASPAR }\end{array}$ & 107 & NS & 44 & "Depressed mood" & 13.1 & $\begin{array}{l}\text { PsAQoL Q4 "I feel there } \\
\text { is no enjoyment in my } \\
\text { life" }\end{array}$ \\
\hline $\begin{array}{l}\text { Davis (2011) } \\
\quad[37]\end{array}$ & $\mathrm{CS}$ & ICD-9 code & 4795 & $52(12)$ & 49 & $\begin{array}{l}\text { Anxiety } \\
\text { Depression } \\
\text { Major depressive disorder } \\
>1 \text { sleep comorbidity }\end{array}$ & $\begin{array}{l}3.9 \\
6.1 \\
3.5 \\
7.6\end{array}$ & ICD-9 codes \\
\hline $\begin{array}{l}\text { Kaine (2019) } \\
\quad[38]\end{array}$ & $\mathrm{L}$ & ICD-9 code & 14,898 & $53(12)$ & 55 & $\begin{array}{l}\text { Anxiety } \\
\text { Depression }\end{array}$ & $\begin{array}{l}9.2 \\
13.3\end{array}$ & ICD-9 codes \\
\hline $\begin{array}{l}\text { Ballegaad } \\
\quad(2017) \text { [39] }\end{array}$ & $\mathrm{L}$ & Physician diagnosis & 1750 & $48(12)$ & 53 & Anxiety or depression & 5.4 & ICD-10 codes \\
\hline
\end{tabular}


Table 1 (continued)

\begin{tabular}{|c|c|c|c|c|c|c|c|c|}
\hline Study & $\begin{array}{l}\text { Study } \\
\text { design }\end{array}$ & PsA definition & $\begin{array}{l}\text { Sample } \\
\text { size }\end{array}$ & $\begin{array}{l}\text { Age, } \\
\text { mean } \\
(\mathrm{SD})\end{array}$ & $\begin{array}{l}\text { Females, } \\
\%\end{array}$ & $\begin{array}{l}\text { Mental health } \\
\text { comorbidity }\end{array}$ & $\begin{array}{l}\text { Prevalence, } \\
\%\end{array}$ & $\begin{array}{l}\text { Criteria or definition } \\
\text { used }\end{array}$ \\
\hline $\begin{array}{l}\text { Altobelli } \\
\text { (2007) [40] }\end{array}$ & $\mathrm{CS}$ & Self-reported & 508 & NS & NS & Depression & 30 & Self-reported \\
\hline $\begin{array}{l}\text { Sinnathurai } \\
\text { (2018) [41] }\end{array}$ & $\mathrm{CS}$ & Physician diagnosis & 490 & $50(12)$ & 59 & Depression & 35.9 & Self-reported \\
\hline $\begin{array}{l}\text { Patel (2018) } \\
\quad[42]\end{array}$ & $\mathrm{CS}$ & $\begin{array}{l}\text { Rheumatologist } \\
\text { diagnosis }\end{array}$ & 164 & 53 & 39 & Depression & 7.1 & $\begin{array}{l}\text { Self-report of receiving } \\
\text { antidepressant }\end{array}$ \\
\hline $\begin{array}{l}\text { Husted } \\
\qquad \text { (2013) [43] }\end{array}$ & $\mathrm{CS}$ & $\begin{array}{l}\text { Physician diagnosis, } \\
\text { 98\% fulfilled } \\
\text { CASPAR }\end{array}$ & 631 & $50(13)$ & 41 & Anxiety or depression & 20.6 & $\begin{array}{l}\text { Patient-reported and } \\
\text { from medical chart } \\
\text { review }\end{array}$ \\
\hline $\begin{array}{l}\text { Khraishi } \\
\text { (2014) [44] }\end{array}$ & $\mathrm{CS}$ & CASPAR & 196 & $49(10)$ & 50 & Anxiety or depression & 13.8 & $\begin{array}{l}\text { Patient-reported and } \\
\text { from medical chart } \\
\text { review }\end{array}$ \\
\hline \multirow[t]{2}{*}{$\begin{array}{l}\text { Löfvendahl } \\
\qquad \text { (2018) [45] }\end{array}$} & \multirow[t]{2}{*}{$\mathrm{CS}$} & \multirow[t]{2}{*}{ ICD-10 code } & \multirow[t]{2}{*}{2657} & \multirow[t]{2}{*}{$55(16)$} & \multirow[t]{2}{*}{56} & $\begin{array}{l}\text { Mental and behavioural } \\
\text { disorders }\end{array}$ & 22.8 & ICD-10 codes \\
\hline & & & & & & $\begin{array}{l}\text { Psychiatric disease including } \\
\text { schizophrenia and mood } \\
\text { disorders }\end{array}$ & 11.3 & ICD-10 codes \\
\hline $\begin{array}{l}\mathrm{Wu}(2016) \\
\quad[46]\end{array}$ & $\mathrm{L}$ & $\begin{array}{l}\text { ICD-9 code and on } \\
\text { biologics }\end{array}$ & 980 & $45(13)$ & 31 & $\begin{array}{l}\text { Depression or insomnia or on } \\
\text { antidepressants }\end{array}$ & 35.6 & ICD-9 codes \\
\hline $\begin{array}{l}\text { Wong }(2017) \\
{[47]}\end{array}$ & $\mathrm{CS}$ & CASPAR & 113 & $57(12)$ & 45 & Poor sleep quality & 84.1 & $\begin{array}{l}\text { Pittsburgh Sleep Quality } \\
\quad \text { Index } \geq 6\end{array}$ \\
\hline $\begin{array}{l}\text { Gezer (2017) } \\
\quad[48]\end{array}$ & $\mathrm{CS}$ & CASPAR & 41 & $41(13)$ & 61 & Poor sleep quality & 85.4 & $\begin{array}{l}\text { Pittsburgh Sleep Quality } \\
\quad \text { Index } \geq 6\end{array}$ \\
\hline
\end{tabular}

\section{Discussion}

Mental health comorbidities are common among patients with PsA. In this systematic review and meta-analysis pooling data of over 30 thousand PsA patients, 1 in 3 screened positive for at least mild anxiety, while 1 in 5 reported at least mild depression. The prevalence of at least moderate anxiety $(21 \%)$ and depression (14\%) was lower on screening. Patients with anxiety and/or depression reported greater disease activity, although the number of studies was small. Sleep problems were also common (84\%) but other mental health comorbidities were rarely reported.
Fig. 1 Forest plots showing the pooled prevalence of anxiety in studies of PsA. The top panel (a) shows prevalence estimates for at least mild anxiety, and bottom panel (b) at least moderate

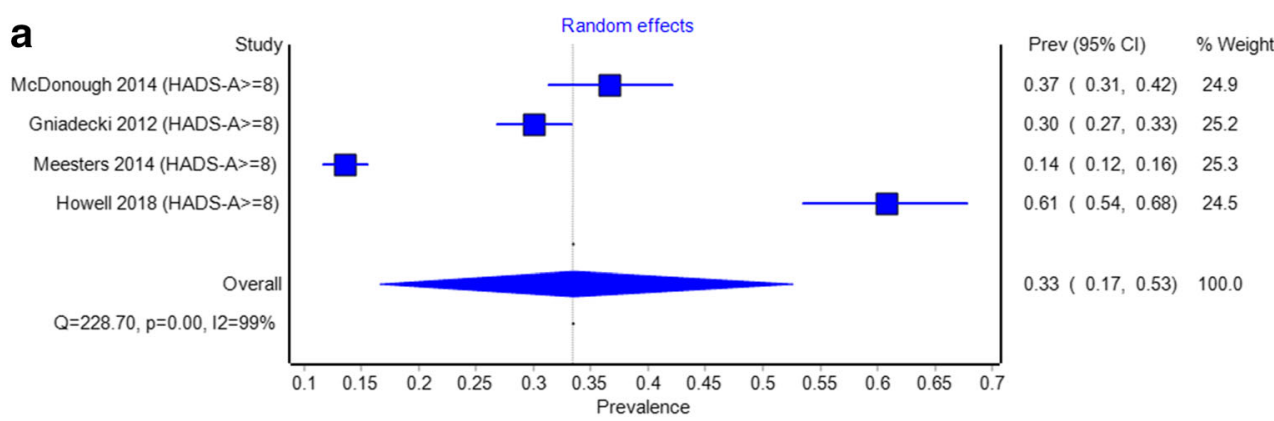

b

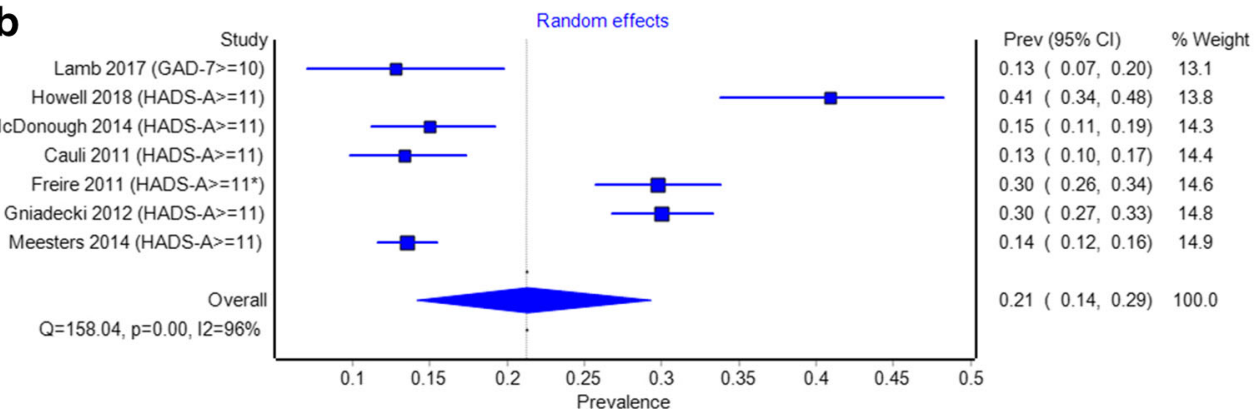


Fig. 2 Forest plots showing the pooled prevalence of depression in studies of PsA. The top panel (a) shows prevalence estimates for at least mild depression, and bottom panel (b) at least moderate

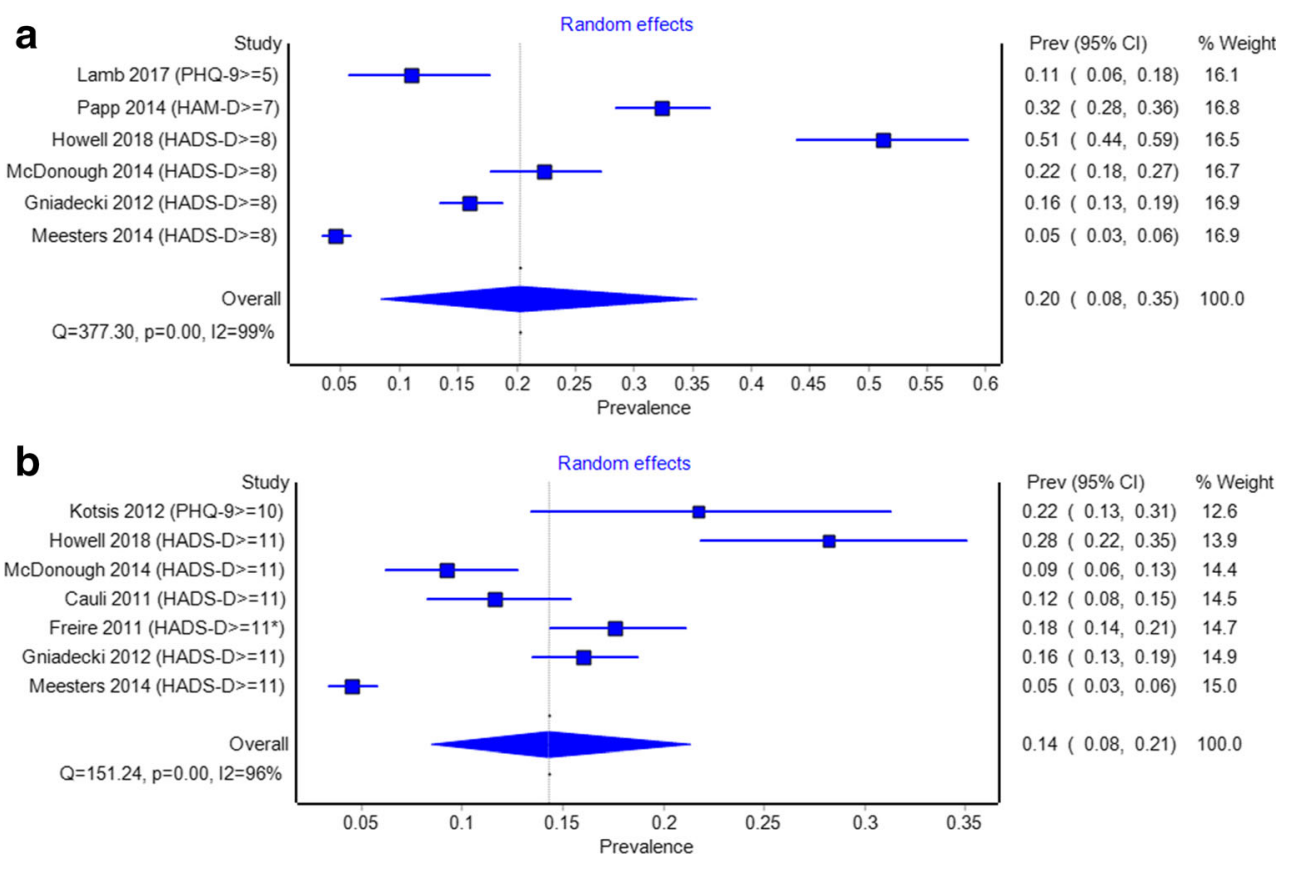

High prevalence of mental health comorbidities among people with psoriasis is well recognised. Features of PsA can be considered additional psoriatic disease burden with accompanying functional impairment. Although metaanalysis estimates for PsA and psoriasis populations cannot be directly compared, several studies have demonstrated higher burden of anxiety and depression in psoriasis patients with PsA than those without: McDonough et al. showed that at least mild anxiety and depression were more prevalent in psoriasis patients with PsA than those without (17.7 vs 6.7\%) [26]. Compared with the general population, the risk of depression in people with psoriasis was $14 \%$ higher, while those with PsA was 22\% higher [49]. Among females, the risk of depression was $29 \%$ higher in those with psoriasis, but $52 \%$ higher if both psoriasis and PsA were present [50].

The prevalence of depression in this meta-analysis (14$20 \%$ ) is similar to those reported in other inflammatory arthritides. A meta-analysis in rheumatoid arthritis (RA) reported at least moderate depression (HADS $\geq 11$ ) in $15 \%$ of patients and at least mild depression (HADS $\geq 8$ ) in $34 \%$ [40]. Virtually identical findings (15\% moderate, $38 \%$ mild) were reported in an axial spondyloarthritis (axSpA) meta-analysis
Fig. 3 The top panel (a) shows prevalence estimates for anxiety or depression, and bottom panel (b) anxiety and depression in studies of PsA

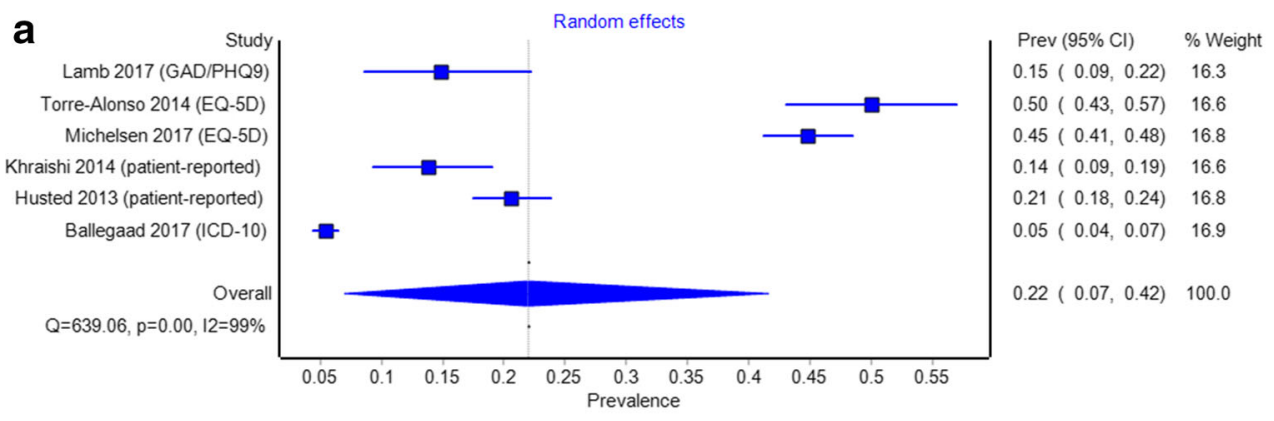

b

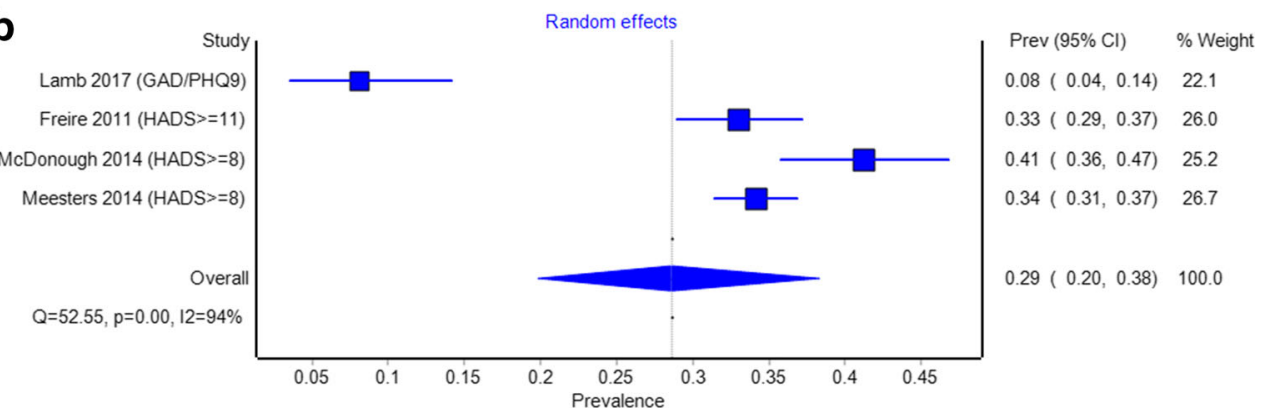


Table 2 Results from two studies comparing psoriatic arthritis patients with and without comorbid anxiety or depression

\begin{tabular}{|c|c|c|c|c|c|}
\hline Study & Mental health disorder & Disease outcome measure & $\begin{array}{l}\text { No mental health } \\
\text { disorder }\end{array}$ & $\begin{array}{l}\text { Mental health disorder } \\
\text { present }\end{array}$ & $P$ value \\
\hline \multirow[t]{6}{*}{ Freire (2011) [30] } & \multirow[t]{3}{*}{ Anxiety (HADS-A $\geq 11$ ) } & DAS28 $(n=341)$ & $2.9 \pm 1.3$ & $3.2 \pm 1.3$ & $<0.05$ \\
\hline & & $\operatorname{BASDAI}(n=41)$ & $6.1 \pm 1.1$ & $9.2 \pm 1.2$ & $>0.05$ \\
\hline & & Pain VAS $(n=321)$ & $3.0 \pm 2.3$ & $4.1 \pm 2.3$ & $<0.01$ \\
\hline & \multirow[t]{3}{*}{ Depression (HADS-D $\geq 11$ ) } & DAS28 $(n=341)$ & $2.9 \pm 1.3$ & $3.3 \pm 1.3$ & $>0.05$ \\
\hline & & $\operatorname{BASDAI}(n=41)$ & $7.4 \pm 1.2$ & $7.1 \pm 1.1$ & $>0.05$ \\
\hline & & Pain VAS $(n=321)$ & $3.1 \pm 2.3$ & $4.3 \pm 2.2$ & $<0.01$ \\
\hline \multirow{11}{*}{$\begin{array}{l}\text { Michelsen (2017) } \\
\text { [35] }\end{array}$} & \multirow{11}{*}{$\begin{array}{l}\text { Anxiety or depression (EQ-5D) } \\
\quad n=728\end{array}$} & DAS28-CRP & $3.7 \pm 1.1$ & $4.0 \pm 1.1$ & 0.002 \\
\hline & & DAS28-ESR & $3.8 \pm 1.2$ & $4.1 \pm 1.3$ & 0.002 \\
\hline & & Pain VAS & $4.2 \pm 2.3$ & $5.3 \pm 2.2$ & $<0.001$ \\
\hline & & 32 tender joint count & 4 (2 to 8$)$ & $5(2-10)$ & 0.002 \\
\hline & & 32 swollen joint count & $3(1-6)$ & $2(1-5)$ & 0.16 \\
\hline & & Patient global assessment & $4.5 \pm 2.3$ & $5.6 \pm 2.2$ & 0.001 \\
\hline & & $\begin{array}{l}\text { Evaluators global } \\
\text { assessment }\end{array}$ & $3.1 \pm 1.6$ & $3.4 \pm 1.6$ & 0.001 \\
\hline & & CRP mg/L & $6(4-155)$ & $7(3-20)$ & 0.70 \\
\hline & & $\mathrm{ESR} \mathrm{mm} / \mathrm{h}$ & $15(8-26)$ & $17(7-30)$ & 0.42 \\
\hline & & SDAI & $13.9(9.6-21.0)$ & $16.5(11.4-23.3)$ & 0.001 \\
\hline & & CDAI & $12.8(8.7-18.9)$ & $14.8(10.6-21.5)$ & 0.001 \\
\hline
\end{tabular}

[51]. These similarities are somewhat unexpected since the proportions of females - who have a higher risk of depression - are very different in these three rheumatic diseases. Other characteristics important for the development of mental health disorders (e.g. age or comorbidity burden) are also likely to differ. In a large case-control study using UK primary care data, the authors adjusted for these confounders and found similar adjusted risk of depression across RA (HR 1.38), AS (HR 1.36), and mild psoriasis (HR 1.30), but higher in severe psoriasis (HR 1.71) [52]. The risk of anxiety appeared higher in AS (1.44) and psoriasis (1.28-1.33) than RA (1.10). No studies examined whether PsA severity was associated with prevalence or incidence of mental health disorders.

Patients with more severe disease may be at higher risk of developing mental health comorbidities, while some mental disorders have been shown to exacerbate the perception of pain [6]. Systemic inflammation may influence neurocognitive functions $[53,54]$. Only two studies compared disease activity between PsA patients with and without mental health comorbidities. Both found significantly higher DAS28 and pain VAS in those with comorbid anxiety or depression, but there were no differences in swollen joint count, ESR or CRP. These cross-sectional studies do not allow us to distinguish the direction of any causal relationship, but the absence of differences in more objective measures of inflammation suggests it is more likely that these comorbidities are influencing reporting of disease severity. Healthcare professional should be mindful of these comorbidities when making treatment decisions based on composite disease scores; screening may be considered for these comorbidities to aid their diagnosis and management. Future studies should validate existing tools, such as the HADS, in patients with inflammatory rheumatic diseases.

One important finding of this review was the paucity of studies on suicide, self-harm, sexual health, substance misuse and other important mental health-related comorbidities. More research is needed in this area, particularly given that one study suggested a higher incidence rate of "any suicidality" in PsA than the general population [49]. Two studies in this review did highlight the importance of future studies of sleep on PsA outcome measures: $84 \%$ of patients screened positive for having poor-quality sleep. Michelsen et al. also reported that comorbid depression and anxiety were negative predictors of PsA remission [35].

A key strength of this review was a large number of studies and patients included. This allowed us to compare prevalence estimated from screening questionnaires against other methods, namely ICD codes. Prevalence of anxiety $(6 \%)$ and depression (9\%) were much lower using ICD codes, likely due to these codes' low sensitivity for mental health diagnoses $[55,56]$. Including a range of study designs can have implications for variations in effect estimates and may contribute to the high heterogeneity observed. However, heterogeneity remained when we stratified meta-analyses by criteria.

The main limitation was the fact that no studies used validated diagnostic criteria for mental health disorders, such as the Structured Clinical Interview for Diagnostic and Statistical Manual of Mental Disorders. It is likely that screening criteria 
over-estimate while ICD codes under-estimate true prevalence. We also include studies that used different definitions of PsA, although it was not feasible to stratify by definitions of both PsA and mental health. This was taken into account during quality assessments. Almost all studies recruited patients from hospitals; these patients are likely to have more severe disease than a primary care sample. Finally, studies included in this review did not consider other comorbidities and PsA disease features. Fibromyalgia, metabolic syndrome and extra-articular manifestations are highly prevalent [7]; future studies should examine how these related conditions interact with mental health comorbidities and influence the management of PsA patients.

\section{Conclusions}

The results of this paper demonstrate that anxiety and depression are highly prevalent in psoriatic arthritis patients. Prevalence estimates are similar to those reported in RA, but some studies suggested a higher burden of anxiety and depression in psoriasis patients with PsA than those without. More research is needed on other mental health comorbidities, particularly sleep, suicide/self-harm and substance misuse. Comorbid anxiety or depression was associated with worse disease activity. Clinicians should be mindful of this when managing PsA patient. Patients with depression should be appropriately referred and managed. This is especially pertinent if depressive symptoms are thought to adversely influence assessments of treatment response.

\section{Compliance with ethical standards}

\section{Disclosures None}

Open Access This article is distributed under the terms of the Creative Commons Attribution 4.0 International License (http:// creativecommons.org/licenses/by/4.0/), which permits unrestricted use, distribution, and reproduction in any medium, provided you give appropriate credit to the original author(s) and the source, provide a link to the Creative Commons license, and indicate if changes were made.

\section{References}

1. Gladman D, Antoni C, Mease P, Clegg D, Nash P (2005) Psoriatic arthritis: epidemiology, clinical features, course, and outcome. Ann Rheum Dis 64(Suppl 2):ii14-ii17

2. Di Carlo M, Becciolini A, Lato V, Crotti C, Favalli EG, Salaffi F (2017) The 12-item psoriatic arthritis impact of disease questionnaire: construct validity, reliability, and interpretability in a clinical setting. J Rheumatol 44(3):279-285

3. Ferreira BIRC, Abreu JLPDC, Reis JPGD, Figueiredo AMDC (2016) Psoriasis and associated psychiatric disorders. J Clin Aesthetic Dermatol 9(6):36-43

4. Eder L, Polachek A, Rosen CF, Chandran V, Cook R, Gladman DD (2017) The development of psoriatic arthritis in patients with psoriasis is preceded by a period of nonspecific musculoskeletal symptoms: a prospective cohort study. Arthritis Rheum 69(3):622-629

5. Kavanaugh A, Gottlieb A, Morita A, Merola JF, Lin C-Y, Birt J et al (2019) The contribution of joint and skin improvements to the health-related quality of life of patients with psoriatic arthritis: a post hoc analysis of two randomised controlled studies. Ann Rheum Dis 21 annrheumdis-2018-215003

6. Bair MJ, Robinson RL, Katon W, Kroenke K (2003) Depression and pain comorbidity: a literature review. Arch Intern Med 163(20): 2433-2445

7. Lee YC, Lu B, Boire G, Haraoui BP, Hitchon CA, Pope JE et al (2013) Incidence and predictors of secondary fibromyalgia in an early arthritis cohort. Ann Rheum Dis 72(6):949-954

8. Duffield SJ, Miller N, Zhao S, Goodson NJ.2018 Concomitant fibromyalgia complicating chronic inflammatory arthritis: a systematic review and meta-analysis. Rheumatology [Internet]. 16 [cited 2019 Feb 7]; Available from: https://doi.org/10.1093/ rheumatology/key112/4996713

9. Prevoo ML, van't Hof MA, Kuper HH, van Leeuwen MA, van de Putte LB, van Riel PL (1995) Modified disease activity scores that include twenty-eight-joint counts. Development and validation in a prospective longitudinal study of patients with rheumatoid arthritis. Arthritis Rheum 38(1):44-48

10. Coates LC, Fransen J, Helliwell PS (2010) Defining minimal disease activity in psoriatic arthritis: a proposed objective target for treatment. Ann Rheum Dis 69(1):48-53

11. Coates LC, Moverley AR, McParland L, Brown S, Navarro-Coy N, O'Dwyer JL et al (2015) Effect of tight control of inflammation in early psoriatic arthritis (TICOPA): a UK multicentre, open-label, randomised controlled trial. Lancet 386(10012):2489-2498

12. Moher D, Liberati A, Tetzlaff J, Altman DG (2009) Preferred reporting items for systematic reviews and meta-analyses: the PRISMA statement. J Clin Epidemiol 62(10):1006-1012

13. Stern AF (2014) The hospital anxiety and depression scale. Occup Med (Lond) 64(5):393-394

14. Kroenke K, Spitzer RL, Williams JB (2001) The PHQ-9: validity of a brief depression severity measure. J Gen Intern Med 16(9):606-613

15. Hamilton M (1960) A rating scale for depression. J Neurol Neurosurg Psychiatry 23:56-62

16. Spitzer RL, Kroenke K, Williams JBW, Löwe B (2006) A brief measure for assessing generalized anxiety disorder: the GAD-7. Arch Intern Med 166(10):1092-1097

17. National Collaborating Centre for Mental Health2009 Depression in adults with a chronic physical health problem: treatment and management. NICE Clinical Guidance 91 London: National Institute for Health and Clinical Excellence. [Internet]. Available from: https://www.nice.org.uk/guidance/CG91

18. Matcham F, Norton S, Steer S, Hotopf M (2016) Usefulness of the SF-36 Health Survey in screening for depressive and anxiety disorders in rheumatoid arthritis. BMC Musculoskelet Disord 17:224

19. McKenna SP, Doward LC, Whalley D, Tennant A, Emery P, Veale DJ (2004) Development of the PsAQoL: a quality of life instrument specific to psoriatic arthritis. Ann Rheum Dis 63(2):162-169

20. Wells A, Shea B, O'Connell D, et al.2014 The Newcastle-Ottawa scale (NOS) for assessing the quality of nonrandomised studies in meta-analyses. [Internet]. Available from: http://www.ohri.ca/ programs/clinical_epidemiology/oxford.asp

21. Barendregt JJ, Doi SA, Lee YY, Norman RE, Vos T (2013) Metaanalysis of prevalence. J Epidemiol Community Health 67(11): 974-978

22. Husted JA, Thavaneswaran A, Chandran V, Eder L, Rosen CF, Cook RJ, Gladman DD (2011) Cardiovascular and other comorbidities in patients with psoriatic arthritis: a comparison with patients with psoriasis. Arthritis Care Res 63(12):1729-1735

23. Husted JA, Tom BD, Farewell VT, Gladman DD (2012) Longitudinal study of the bidirectional association between pain 
and depressive symptoms in patients with psoriatic arthritis. Arthritis Care Amp Res 64(5):758-765

24. Khraishi M, MacDonald D, Rampakakis E, Vaillancourt J, Sampalis JS (2011) Prevalence of patient-reported comorbidities in early and established psoriatic arthritis cohorts. Clin Rheumatol 30(7):877-885

25. Cauli A, Gladman DD, Mathieu A, Olivieri I, Porru G, Tak PP et al (2011) Patient global assessment in psoriatic arthritis: a multicenter GRAPPA and OMERACT study. J Rheumatol 38(5):898-903

26. McDonough E, Ayearst R, Eder L, Chandran V, Rosen CF, Thavaneswaran A, Gladman DD (2014) Depression and anxiety in psoriatic disease: prevalence and associated factors. J Rheumatol 41(5):887-896

27. Gniadecki R, Robertson D, Molta CT, Freundlich B, Pedersen R, Li W, Boggs R, Zbrozek AS (2012) Self-reported health outcomes in patients with psoriasis and psoriatic arthritis randomized to two etanercept regimens. J Eur Acad Dermatol Venereol JEADV 26(11):1436-1443

28. Meesters JJL, Petersson IF, Bergman S, Haglund E, Jacobsson LTH, Bremander A (2014) Sociodemographic and disease-related factors are associated with patient-reported anxiety and depression in spondyloarthritis patients in the Swedish SpAScania cohort. Clin Rheumatol 33(11):1649-1656

29. Howells L, Chisholm A, Cotterill S, Chinoy H, Warren RB, Bundy C (2018) Impact of disease severity, illness beliefs, and coping strategies on outcomes in psoriatic arthritis. Arthritis Care Res 70(2):295-302

30. Freire M, Rodríguez J, Möller I, Valcárcel A, Tornero C, Díaz G, Armendáriz Y, Paredes S (2011) Prevalence of symptoms of anxiety and depression in patients with psoriatic arthritis attending rheumatology clinics. Reumatol Clin 7(1):20-26

31. Lamb RC, Matcham F, Turner MA, Rayner L, Simpson A, Hotopf M, Barker JNWN, Jackson K, Smith CH (2017) Screening for anxiety and depression in people with psoriasis: a cross-sectional study in a tertiary referral setting. Br J Dermatol 176(4):1028-1034

32. Kotsis K, Voulgari PV, Tsifetaki N, Machado MO, Carvalho AF, Creed F, Drosos AA, Hyphantis T (2012) Anxiety and depressive symptoms and illness perceptions in psoriatic arthritis and associations with physical health-related quality of life. Arthritis Care Res 64(10):1593-1601

33. Papp K, Poulin Y, Vieira A, Shelton J, Poulin-Costello M (2014) Disease characteristics in patients with and without psoriatic arthritis treated with etanercept. J Eur Acad Dermatol Venereol JEADV 28(5):581-589

34. Torre-Alonso JC, Gratacós J, Rey-Rey JS, Valdazo de Diego JP, Urriticoechea-Arana A, Daudén E et al (2014) Development and validation of a new instrument to measure health-related quality of life in patients with psoriatic arthritis: the VITACORA-19. J Rheumatol 41(10):2008-2017

35. Michelsen B, Kristianslund EK, Sexton J, Hammer HB, Fagerli KM, Lie E, Wierød A, Kalstad S, Rødevand E, Krøll F, Haugeberg G, Kvien TK (2017) Do depression and anxiety reduce the likelihood of remission in rheumatoid arthritis and psoriatic arthritis? Data from the prospective multicentre NOR-DMARD study. Ann Rheum Dis 76(11):1906-1910

36. Walsh JA, McFadden ML, Morgan MD, Sawitzke AD, Duffin KC, Krueger GG et al (2014 Aug) Work productivity loss and fatigue in psoriatic arthritis. J Rheumatol 41(8):1670-1674

37. Davis JA, Robinson RL, Le TK, Xie J (2011) Incidence and impact of pain conditions and comorbid illnesses. J Pain Res 4:331-345

38. Kaine J, Song X, Kim G, Hur P, Palmer JB (2019) Higher incidence rates of comorbidities in patients with psoriatic arthritis compared with the general population using U.S. administrative claims data. J Manag Care Spec Pharm 25(1):122-132

39. Ballegaard C, Højgaard P, Dreyer L, Cordtz R, Jørgensen TS, Skougaard M, Tarp S, Kristensen LE (2018) Impact of comorbidities on tumor necrosis factor inhibitor therapy in psoriatic arthritis: a population-based cohort study. Arthritis Care Res 70(4):592-599
40. Altobelli E, Maccarone M, Petrocelli R, Marziliano C, Giannetti A, Peris K, Chimenti S (2007) Analysis of health care and actual needs of patients with psoriasis: a survey on the Italian population. BMC Public Health 7:59

41. Sinnathurai P, Buchbinder R, Hill C, Lassere M, March L (2018) Comorbidity in psoriatic arthritis and rheumatoid arthritis: comorbidity in inflammatory arthritis. Intern Med J 48(11):1360-1368

42. Patel P, Rosen CF, Chandran V, Ye YJ, Gladman DD (2018) Addressing comorbidities in psoriatic disease. Rheumatol Int 38(2):219-227

43. Husted JA, Thavaneswaran A, Chandran V, Gladman DD (2013) Incremental effects of comorbidity on quality of life in patients with psoriatic arthritis. J Rheumatol 40(8):1349-1356

44. Khraishi M, Aslanov R, Rampakakis E, Pollock C, Sampalis JS (2014) Prevalence of cardiovascular risk factors in patients with psoriatic arthritis. Clin Rheumatol 33(10):1495-1500

45. Löfvendahl S, Jöud A, Petersson IF, Theander E, Svensson Å, Carlsson KS (2018 Apr) Income disparities in healthcare use remain after controlling for healthcare need: evidence from Swedish register data on psoriasis and psoriatic arthritis. Eur J Health Econ 19(3):447-462

46. Wu CY, Chang YT, Juan CK, Shen JL, Lin YP, Shieh JJ et al (2016) Depression and insomnia in patients with psoriasis and psoriatic arthritis taking tumor necrosis factor antagonists. Med U S 95(22)

47. Wong ITY, Chandran V, Li S, Gladman DD (2017) Sleep disturbance in psoriatic disease: prevalence and associated factors. J Rheumatol 44(9):1369-1374

48. Gezer O, Batmaz İ, Sariyildiz MA, Sula B, Ucmak D, Bozkurt M, Nas K (2017) Sleep quality in patients with psoriatic arthritis. Int J Rheum Dis 20(9):1212-1218

49. Wu JJ, Penfold RB, Primatesta P, Fox TK, Stewart C, Reddy SP, Egeberg A, Liu J, Simon G (2017) The risk of depression, suicidal ideation and suicide attempt in patients with psoriasis, psoriatic arthritis or ankylosing spondylitis. J Eur Acad Dermatol Venereol 31(7):1168-1175

50. Dommasch ED, Li T, Okereke OI, Li Y, Qureshi AA, Cho E (2015) Risk of depression in women with psoriasis: a cohort study. Br J Dermatol 173(4):975-980

51. Zhao S, Thong D, Miller N, Duffield SJ, Hughes DM, Chadwick L et al (2018) The prevalence of depression in axial spondyloarthritis and its association with disease activity: a systematic review and meta-analysis. Arthritis Res Ther 20(1)

52. Dregan A, Matcham F, Harber-Aschan L, Rayner L, Brailean A, Davis K, Hatch S, Pariante C, Armstrong D, Stewart R, Hotopf M (2019 May 1) Common mental disorders within chronic inflammatory disorders: a primary care database prospective investigation. Ann Rheum Dis 78(5):688-695

53. Chimenti MS, Caso F, Alivernini S, De Martino E, Costa L, Tolusso B et al (2019 Jun) Amplifying the concept of psoriatic arthritis: the role of autoimmunity in systemic psoriatic disease. Autoimmun Rev 18(6):565-575

54. Zunszain PA, Hepgul N, Pariante CM (2013) Inflammation and depression. Curr Top Behav Neurosci 14:135-151

55. Fiest KM, Jette N, Quan H, St. Germaine-Smith C, Metcalfe A, Patten SB, et al. Systematic review and assessment of validated case definitions for depression in administrative data. BMC Psychiatry [Internet]. 2014 Oct 17 [cited 2019 Apr 17];14. Available from: https:/www.ncbi.nlm.nih.gov/pmc/articles/PMC4201696/

56. Kim HM, Smith EG, Stano CM, Ganoczy D, Zivin K, Walters H, Valenstein M (2012 Jan 23) Validation of key behaviourally based mental health diagnoses in administrative data: suicide attempt, alcohol abuse, illicit drug abuse and tobacco use. BMC Health Serv Res 12:18

Publisher's note Springer Nature remains neutral with regard to jurisdictional claims in published maps and institutional affiliations. 